\title{
RFID Enabled Health Monitoring System for Aircraft Landing Gear
}

\author{
Shuai Yang ${ }^{1}$, Student member, IEEE, Michael Crisp, Member, IEEE, \\ Richard V. Penty, Senior Member, IEEE, and Ian H. White, Fellow, IEEE \\ Electrical Division, Department of Engineering, University of Cambridge, Cambridge, United Kingdom \\ ${ }^{1}$ sy313@cam.ac.uk
}

\begin{abstract}
RFID has been used in the aviation industry to track and identify emergency equipment and other in-cabin assets on commercial aircraft for some time. Recently, the industry is looking to expand the use of RFID to more demanding parts and surfaces both inside and outside of an aircraft's cabin, where RFID tags face much harsher conditions. The landing gear (LG) is one of the critical subsystems of an aircraft that plays an essential role in dispersing the energy of landing events and taxiing. Health monitoring of the LG has been suggested to help reduce both operational and maintenance costs, and extend the life of the LG beyond its current, fixed, designed service life. In this paper, we propose a health monitoring system using a combination of active wired sensors and passive RFID tags. We present the measurement of Ultra-high Frequency (UHF) RFID tags on an aircraft landing gear using an aircraft-mounted fixed RFID reader. The results indicate that all major landing gear components and assemblies are shown to be identifiable by their EPC, and a $7 \mathrm{~dB}$ system margin has been achieved using 2 RFID reader antennas. Such a margin will tolerate degradations caused by harsh environments (e.g. low temperatures and high humidity) and enable update of information (e.g. flight count) stored on the RFID tags.
\end{abstract}

Keywords-landing gear; health monitoring; configuration control; UHF; RFID; aircraft; aviation.

\section{INTRODUCTION}

Radio Frequency Identification (RFID) has been widely used in various industrial applications for purposes such as access control, fraud prevention and inventory management [1]. The adoption of this technology in different domains has commonly resulted in significant improvements in operational efficiency beyond those using barcodes. In the aviation industry, the early deployment of RFID technology was to track and locate luggage, cargo and containers [2-5]. A number of trials and case studies conducted by airports, airlines and aircraft manufacturers have suggested that the adoption of the RFID technology in the aviation industry can improve management efficiency and enhance safety and quality control, bringing enormous benefits for both airlines and passengers [67].

Recently, the need for accurate tracking of aircraft parts and maintaining relevant operational information has opened new applications for RFID. Major manufacturers in the aviation industry have been working together to develop common industry standard solutions for RFID on commercial aircraft parts [8]. While RFID systems have allowed significant reductions in inspection and maintenance time, to date they have mainly focused on in-cabin assets and interior parts of an aircraft with tags being used as direct replacements for nameplates. The reading of tags has normally been carried out by hand.

In this paper, we demonstrate how passive UHF RFID technology can be used to improve health monitoring of an aircraft landing gear (LG). An RFID-based configuration control system that can wirelessly collect LG parts information stored on the RFID tags is introduced. To the best of the authors' knowledge, this is the first publication of its kind to detail proof of concept results using fixed readers for external on-aircraft applications.

The rest of the paper is organised as follows. The architecture for the RFID landing gear health monitoring system and its control are introduced in Section II. Section III provides details on component selections and measurements of their characteristics. Section IV presents a model for the RFID system based on the measured radiating characteristics of the RFID tags and reader antennas to determine the system feasibility. Section V provides details on the aircraft-level tests and analysis of results. Finally, conclusions are drawn in Section VI.

\section{RFID-ENABLED LG HEALTH MONITORING SYSTEM}

\section{A. Landing Gear Health Monitoring System}

An aircraft landing gear is a critical subsystem of an aircraft and plays an essential role in dispersing the energy of landing events and taxiing. Health monitoring of landing gear would enable a reduction in maintenance costs, extending the life of landing gear beyond the current service life without compromising safety. An ideal structural health monitoring system would be able to detect changes within the material of the LG components and accurately predict failures such that the components can be taken out of service before failure occurs. Current sensor technology is unable to detect such degradation. Therefore, it is proposed to monitor the stresses encountered by each component over its historic operating time, to enable predictions of its remaining safe service lifetime. However, it is essential from a certification and safety point of view that there 
is complete traceability between the recorded data and components in use at the time of recording. Similar systems have been proposed for use on Rotorcraft [9-11]. However, to the authors knowledge detailed measurement studies on the use of RFID for health and usage monitoring system (HUMS) in landing gear have not been carried out.

To enable the remaining lifetime prediction of the whole landing gear, the HUMS must check the condition of the landing gear at the component level, with each component being associated with its manufacturing date, (predicted) wear level, and its usage and maintenance history. Due to the intensive stresses experienced, the LG is likely to be overhauled regularly within the lifespan of the airframe. During overhaul, it is entirely possible for components originating from different landing gears to be swapped into new assemblies. As a result, the health monitoring system must be able to ensure the tracking of all component changes and be robust to maintenance performed without proper records being kept.

Currently, the wear caused by each landing must be assessed by measurements from wired sensors placed on the gear itself. However, in future the loads on landing gear components may be estimated from flight recorder data using machine learning techniques [12]. The algorithms for predicting wear from the sensor data are beyond the scope of this paper.

Fig.1 (a) shows the simplified system architecture of the proposed landing gear health monitoring system which

(a)

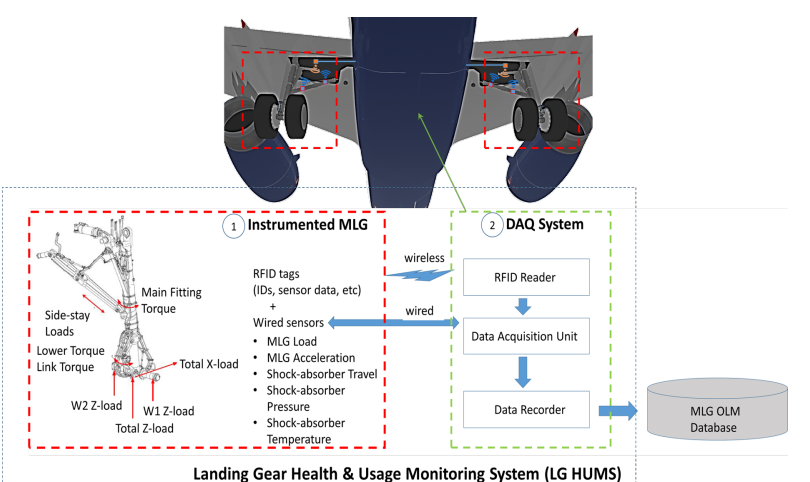

Landing Gear Health \& Usage Monitoring System (LG HUMS)

(b)

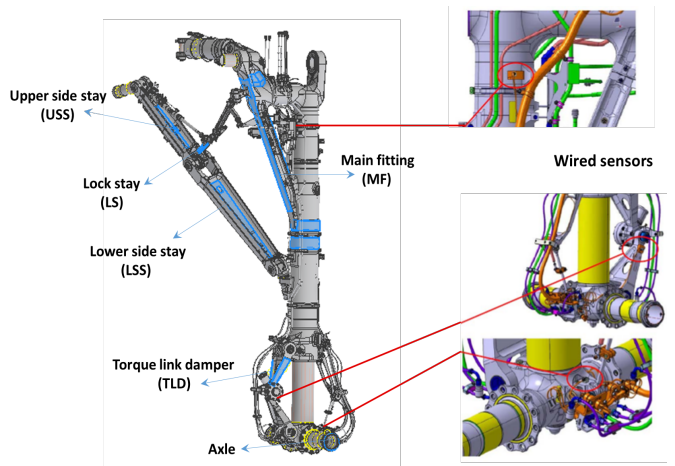

Fig. 1 The proposed landing gear health monitoring system. (a) the system consists of two major parts: two instrumented main landing gears (MLGs), and a data acquisition system (DAQ) that contains an RFID reader. (b) a main landing gear instrumented with multiple RFID tags and wired sensors (several types of sensors are installed but only the obvious ones are shown here). The LG model supplied courtesy of Safran Landing Systems UK Limited comprises of two main parts: the two main landing gears (MLGs) which are instrumented with a set of wired sensors and RFID tags; and a data acquisition system (DAQ, located in the LG bay) with an integrated RFID reader to record and store tag and sensor data. Fig. 1 (b) shows an instrumented MLG with RFID tags placed on the major sub-assemblies (parts highlighted in blue). Each tag uniquely identifies the serial number of subassembly it is attached to. The tags also store the calibration parameters of the wired sensors on the sub-assembly to allow the recorded sensor data to be accurately translated into the physical parameters which the LG has experienced. Finally, critical information of each LG component since its manufacture, such as flight count and previous important maintenance /inspection histories is stored. A fixed RFID reader is installed within the DAQ, allowing the tags to be interrogated after each landing when the aircraft is stationary. This allows the configuration of the landing gear and the calibration parameters to be checked and appended to the data from each take-off / landing cycle when the aircraft is parked and stationary (avoiding strict regulatory requirements for RF transmitters in flight). By storing the data in this way, even if unscheduled and un-notified maintenance is carried out, the health monitoring system is able to apply the correct calibration factors to the recorded data and attribute the wear to the correct components.

At periodic intervals information stored in the DAQ can be exported to an external Operational Loads Monitoring (OLM) database for post processing and data analysis.

\section{B. RFID-based Configuration Control System}
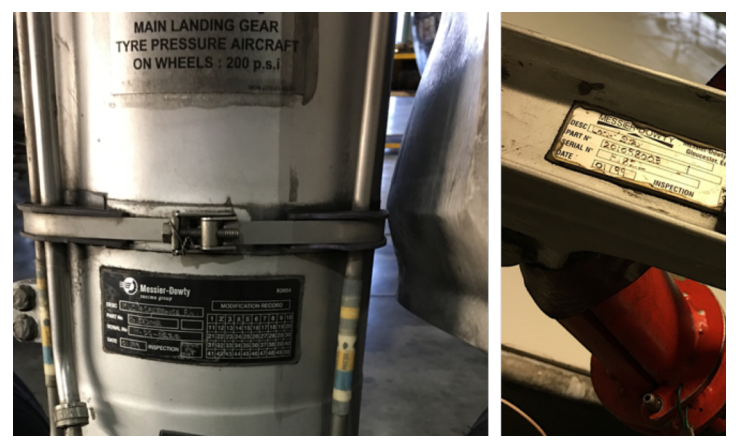

Fig. 2 Nameplate-based traditional aircraft parts marking: MLG main fitting (left), and lock stay (right)

As shown in Fig. 2, current LG part marking is based on a metal nameplate attached to the part. Information such as the part number, serial number, and manufacturing date are either laser printed or chemically etched in a human readable format. In addition, a separate modification plate (the black metal plate in Fig.2) is usually used to record the component modification history. While the nameplate is very robust, only a limited amount of data can be stored. In addition, for some LG components such as the side stays and lock stays, the nameplates are often obscured from direct view so the configuration of the landing gear is usually only checked at major service intervals.

Passive UHF RFID tags are proposed to be used in conjunction with nameplates to improve the visibility and traceability of the components. Fig. 3 depicts the simplified RFID system architecture and some major LG components to be tracked. Clearly, it is essential that the system is able to detect 


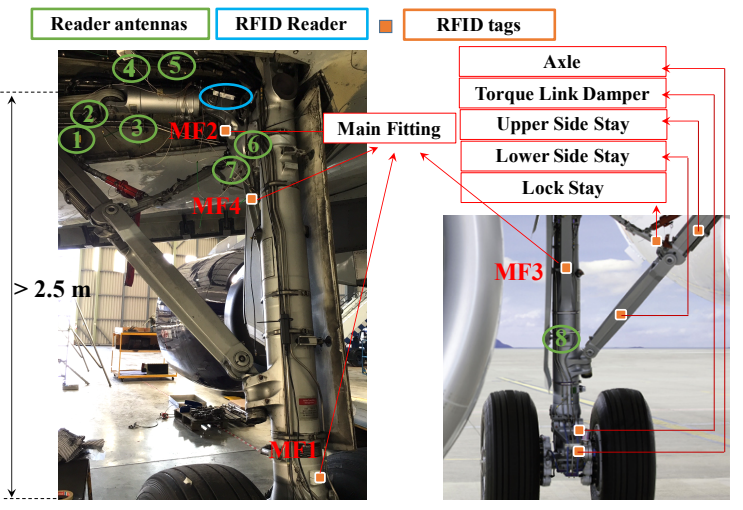

Fig. 3 RFID system architecture and locations of link components. The RFID reader location (inside the blue circle) shown here is only to ease the connections to the reader antennas. In real implementation the reader is integrated into the DAQ which is placed in the LG bay

successfully all the tagged LG components. Since the tags have no on-board power source and can only derive their operating power from the reader transmitter, the locations of the reader antennas become extremely important. When the landing gear is down, the wing bay door remains open, providing a line-of-sight (LoS) to the landing gear from the bay, making the bay an attractive location for the reader antennas. Due to the long distances between tags and antennas (e.g. $>2.5 \mathrm{~m}$ between the LG axle and the wing bay), and possible screening due to objects in or close to the the LG axle and the wing bay, suitable locations on the main fitting are also considered. Further issues exist as the system operates in a metal-rich environment as alloys are used in most components of the LG due to their superior strength and stiffness properties. The complex structural properties of the LG and the wing bay provide a challenging operating environment for the passive RFID system.

\section{SYSTEM COMPONENT SELECTIONS AND CHARACTERISATION}

\section{A. Selections of RFID tags and reader antennas}

In passive UHF RFID systems, the maximum downlink (i.e. reader-to-tag link) and uplink (i.e. tag-to-reader link) operating ranges $D_{\max , d}$ and $D_{\max , u}$ can be estimated by:

$$
\begin{aligned}
& D_{\text {max }, d}=\sqrt{\frac{P_{\text {reader }, T} \cdot G_{\text {reader }, T, \text { max }} \cdot G_{\text {tag }, R, \text { max }} \cdot C^{2} \cdot X_{d} \cdot \tau}{(4 \pi f)^{2} \cdot L_{d b} \cdot P_{I C}}}, \\
& D_{\text {max }, u}=\sqrt{\frac{P_{\text {tag,re-radiated }} \cdot G_{\text {tag }, T, \text { max }} \cdot G_{\text {reader }, R, \text { max } \cdot C^{2} \cdot X_{u}}}{(4 \pi f)^{2} \cdot L_{u b} \cdot P_{\text {reader }}}},
\end{aligned}
$$

where $P_{\text {reader, } T}$ is the output power (before the reader antenna) of the RFID reader transmitter, and $G_{\text {reader,T,max }}$ is the peak gain of the reader transmit antenna. The product of the two determines the total reader radiated power that must be limited to certain values to meet various local regulations. $G_{\text {reader, }, \text { max }}$ is the peak gain of the reader receive antenna, $G_{\text {tag,T,max }}$ is the peak gain of the tag transmit antenna, and $G_{t a g, R, \max }$ is the peak gain of the tag receive antenna. Gains of reader and tag antennas are functions of carrier frequencies and their relative orientations (angles). $c$ is the speed of light, $X_{d}$ is the downlink polarisation mismatch loss, $X_{u}$ is the uplink polarisation mismatch loss, $f$ is the carrier frequency, $L_{d b}$ is the downlink blockage loss, $L_{u b}$ is the uplink blockage loss, and $\tau$

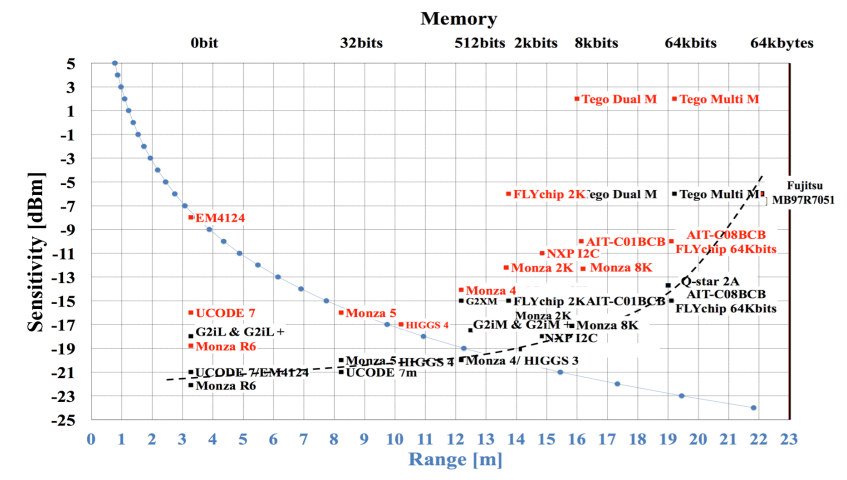

Fig. 4 A survey of COTS RFID ICs. Fonts in black and red represent the ICs' read and write sensitivities respectively. The top horizontal axis represents the memory capacities of the ICs underneath, the bottom horizontal axis represents the theoretical read and write ranges of the ICs

is the power transmission coefficient between the tag antenna and IC. $P_{\text {tag_re-radiated }}$ is the reader power backscattered towards the reader receiver by the tag. $P_{I C}$ and $P_{\text {reader }}$ are the tag IC and reader receiver sensitivities. In the absence of surrounding reflections, the maximum range is achieved when the peak gains of reader and tag antennas are facing each other. In a monostatic RFID system, since the antenna is used for both transmitting and receiving purposes, the blockage and mismatch losses in the downlink and uplink are the same. In addition, the transmit and receive gains of reader and tag antennas are also identical according to antenna reciprocity.

In most cases the receiver sensitivity (and hence the maximum uplink range) of a bistatic RFID system is better than a monostatic RFID system. However, the downlink and uplink losses (e.g. blockage loss) in a bistatic RFID system are often different, which may result in a higher cost but poorer performance than a monostatic RFID system. The difference could be more obvious when directional antennas are used by both RFID readers and tags.

An extensive survey of commercial-off-the-shelf (COTS) passive UHF RFID IC has been carried out and the ICs' sensitivities (i.e. read and write) together with their theoretical maximum range (blue dotted curve) are plotted in Fig. 4. The theoretical range is calculated using Eq. 1 and assumes that the system operates in the lower ETSI frequencies $(865-868 \mathrm{MHz})$ with a reader transmitted power of $35 \mathrm{dBm}$ EIRP via a circular polarised antenna (hence a $3 \mathrm{~dB}$ polarisation mismatch loss). In addition, the tag antenna is assumed to have a gain of $2 \mathrm{dBi}$, and no mismatch loss exists between the tag IC and its antenna (i.e. $\tau=1$ in Eq. 1).

In addition to the operating range requirement, the tags must also provide sufficient memory capacity for the storage of LG part information and sensor calibration data. Currently, most tags on the market have a user memory of 512 bits or less. These tags have a theoretical maximum range over $10 \mathrm{~m}$ which is sufficient for most applications, such as logistics and asset management. The write range is somewhat lower, however, most RFID applications only require knowledge of the 'existence' of the asset, and thus the write sensitivity and the IC memory capacity are usually less important. This is not the case for the HUMS system which requires significant additional details to be stored and records regularly updated. 
TABLE I CHARACTERISTICS OF THE SELECTED TAGS AND READER ANTENNAS

\begin{tabular}{|c|c|c|c|c|}
\hline \multicolumn{5}{|c|}{ RFID tags } \\
\hline$T a g$ & IC & Typical read/write sensitivity [dBm] & Memory capacity /kbits] & Dimensions [cm] \\
\hline Omni-ID $[15]$ & Q-star 2A $\left[{ }^{[17]}\right.$ & $-14 /$ (not mentioned) & 64 & $2.5 \times 2.5 \times 0.6$ \\
\hline$H I_{[\text {[1.6] }}$ & Impinj Monza 8K ${ }^{[18]}$ & $-17 /-12$ & 8 & $2 \times 4 \times 4.5$ \\
\hline \multicolumn{5}{|c|}{ RFID reader antennas } \\
\hline & Dimensions $[\mathrm{cm}]$ & \multicolumn{2}{|l|}{ Peak gain [dBi] } & Polarisation \\
\hline & $5 \times 5$ & \multicolumn{2}{|l|}{2.5} & Circular polarised \\
\hline
\end{tabular}

The ATA Spec 2000 standard [13] has defined three types of tags for aircraft component tracking applications according to the available tag memory capacity: single-record (>1 kbits), dual-record ( $>2$ kbits), and multi-record ( $>4$ kbytes). For the proposed application, dual-record or multi-record RFID tags are necessary to enable the storage of the calibration data for multiple sensors, as well as from the birth record and maintenance history for each LG component. The greater the memory capacity, the more the critical information can be stored. However, a general trend can be observed from the black dotted curve in Fig. 4, that as the IC's memory capacity increases the IC's read and write sensitivities become poorer, and in turn, the IC's maximum ranges decrease. To date the maximum available memory capacity is 64 kbytes (Fujitsu MB97R7051 [14]), however according to the blue dotted curve, its low sensitivity of $-6 \mathrm{dBm}$ leads to a theoretical maximum read range less than $3 \mathrm{~m}$. Ideally this IC meets both the range (marginally meet) and memory requirements. However the calculation here neglects the effects of nearby objects (e.g. distortions of tag antenna) which always exist in practical applications. In addition it also brings challenges in tag antenna design and the placement selections of tag and reader antennas.

Based on the memory requirement of $\sim 150$ bytes for each sensor with an efficient binary encoding scheme, and the operating range requirement $>2.5 \mathrm{~m}$, two COTS metal mountable RFID tags: Omni-ID Adept 850 tag [15] and HL tag [16] were selected as the identification carrier to be affixed on to the LG components. The Q-star 2A [17] and Impinj Monza $8 \mathrm{~K}$ [18] ICs are used respectively by the tags. According to the blue dotted curve in Fig. 4, ideally tags with these ICs can offer a range greater than $7 \mathrm{~m}$ which meets the system range requirement. However the actual range depends on the antenna design. The Omni-ID tag, uses a "plasmonic structure" [15] to decouple the tag from any attached and nearby objects, including liquids. Ceramic is used as the substrate and therefore provides a small but robust structure. In addition the antenna supports both ETSI and FCC frequency bands and can be used globally. The HL tag on the other hand uses simple folded wire dipole which is spaced away from any metal by the physical size of the tag and only supports the lower ETSI frequency band.

A set of $5 \mathrm{~cm}$ x $5 \mathrm{~cm}$ circular polarised ceramic antennas were selected to be installed in the wing bay and on the main fitting due to their low profiles and compact size which enable the use of the pipes running through the wing bay as the mounting points. Characteristics of the selected RFID tags and reader antennas are listed in Table I.

\section{B. Characteristics of RFID tags and reader antennas}

Although antennas and tags often specify the maximum gain or sensitivity under specific test conditions, few manufacturers (a)

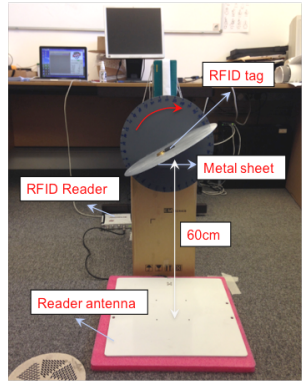

(b)
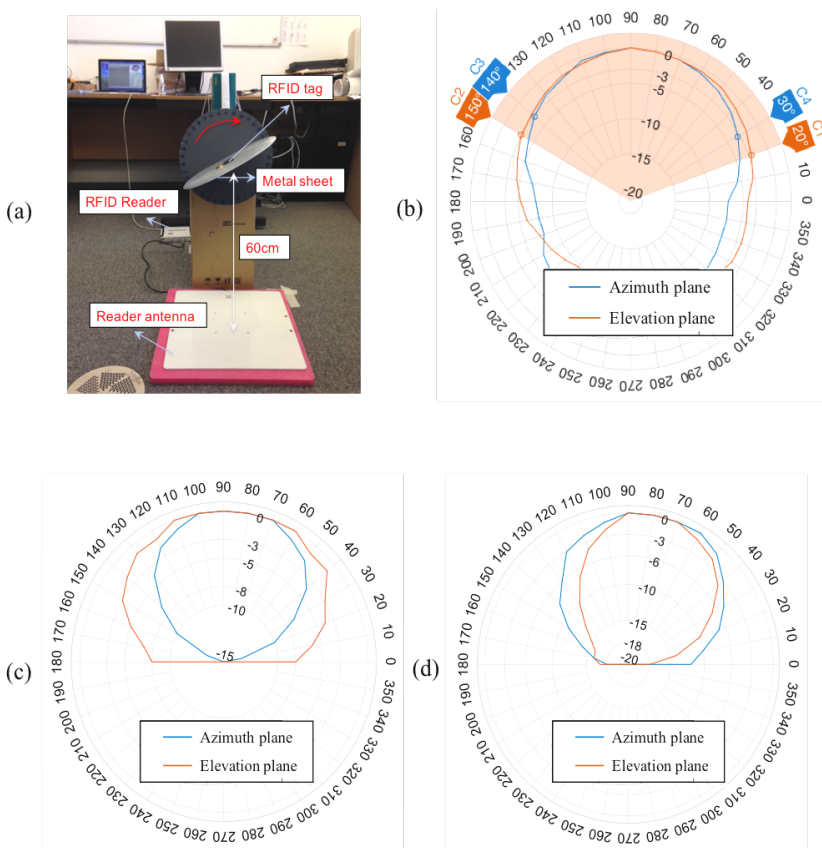

Fig. 5 (a) Test setup (b) radiation patterns of the reader antenna (c) radiation patterns of Omni-ID Adept 850 tag (d) radiation patterns of HL tag

supply detailed radiation patterns which are required to allow accurate prediction of the range in less than ideal circumstances. Ideally, the measurements of radiating characteristics of reader antennas and tags shall be performed in an anechoic chamber to reduce effects caused by surrounding reflections. However, it has been suggested by [19] that acceptable results can be obtained by placing the tag or reader antenna under test $0.5-1 \mathrm{~m}$ away from the test equipment, and as far as possible from nearby reflectors. Fig. 5 (a) shows the experimental setup to determine the maximum read range of the two tags. The tags are mounted on a circular metal ground plane $(30 \mathrm{~cm}$ in diameter) and measured $60 \mathrm{~cm}$ away from a reader antenna. Clearly the relatively small ground plane can have some effect on the measured pattern, however it is expected to be representative of a tag mounted on a reasonably large metal object as found on the landing gear. It will also effect the return loss of the reader antenna, and potentially the reader sensitivity, but by using a short transmission distance we can ensure that our system is forward link limited. By gradually increasing the reader transmitted power, the tag threshold can be determined and the tag's maximum read range $D_{\max }$ can be estimated by [20]:

$$
D_{\text {max }}=d \sqrt{\frac{E I R P}{P_{\text {ref }} G_{\text {reader }} L_{\text {cble }}}},
$$

where in our case $d=0.6 \mathrm{~m}$ is the reference distance, $G_{\text {reader }}$ is the gain of the reader antenna, $L_{\text {cable }}$ is the loss of the RF cable, EIRP is $3.2 \mathrm{~W}$ for RFID applications operate in the lower ETSI bands (865-868 MHz) [21-22], and $P_{r e f}$ is the minimum required reader transmitted power (before the reader antenna) to detect the tag.

The radiation patterns of the tags can be determined by rotating them with respect to their axes and recording each $P_{\text {ref }}$ at each angle. In a similar manner, the reader antenna's radiation patterns can also be plotted, but a spectrum analyser is needed 
to determine the received power at each angle. When installed on the LG components the tags are likely facing the reader antenna, and hence only the edge to edge radiation patterns were measured for the tag antennas. The reader antennas' and tags' radiation patterns are plotted in figures 5 (b), 5 (c), and 5 (d) respectively.

To ease the presentation of each antenna's $3 \mathrm{~dB}$ beamwidths, all the antenna gains are normalised to the peak gains and values shown are in the logarithmic scale. It can be seen that the radiation patterns of both reader and tag antennas are reasonably symmetric with peak gains in the centre. For the reader antenna, its $3 \mathrm{~dB}$ beamwidth is wider than $90^{\circ}$ in both azimuth and elevation planes. Under ETSI regulations [21], such a broad beamwidth results in the maximum allowable reader transmitted power being reduced by $3 \mathrm{~dB}$ to $1 \mathrm{~W}$ ERP (or $32.15 \mathrm{dBm}$ EIRP). Nonetheless, it is still expected that using an antenna with a wide beamwidth would be advantageous, since the geometric properties of the LG (see Fig. 3) cause the tags to be set at a wide range of angles with respect to any antenna. It is especially important for those antenna locations where tags close to the edge of the field of view will be facing the reader antennas edge on and hence have a poor gain. According to the measured radiation patterns shown in Fig. 5, at least $8 \mathrm{~dB}$ reduction in gain can be found for both tags, when reading the tags edge on.

By utilising Eq. 3 the maximum read ranges for Omni-ID tag and $\mathrm{HL}$ tag were estimated to be $4 \mathrm{~m}$ and $7.6 \mathrm{~m}(\sim 5.5 \mathrm{~dB}$ difference in $\left.P_{\text {ref }}\right)$ respectively. Although the different antenna designs may contribute part of the difference, it is mainly the lower IC sensitivity (see Fig. 4 and Table I) that leads to the shorter range of the Omni-ID tag. However, HL tag is shown to have narrower radiation patterns (i.e. its gains fall off quicker towards the edges), and therefore it is not expected that HL tag will perform better on all LG components. In addition, one attractive feature of Omni-ID tag is that its antenna supports both the lower ETSI band (865-868 MHz) and the FCC band (including the upper ETSI band (915-921 MHz)), and the performance difference between the two bands is measured and shown in Fig. 6. It can be seen that, the Omni-ID tag requires 4 $\mathrm{dB}$ less power when it operates in the upper ETSI band. Considering that the regulations allow the reader to transmit 3 $\mathrm{dB}$ more power in the upper band $[21,22]$, we would expect that the performance of Omni-ID tag in the upper ETSI band will be similar to or better than that of HL tag in the lower ETSI band $(7 \mathrm{~dB}>5.5 \mathrm{~dB}$, here assumes the reader antennas have identical characteristics in both ETSI bands). For monitoring LG components, worldwide operation is highly desirable which

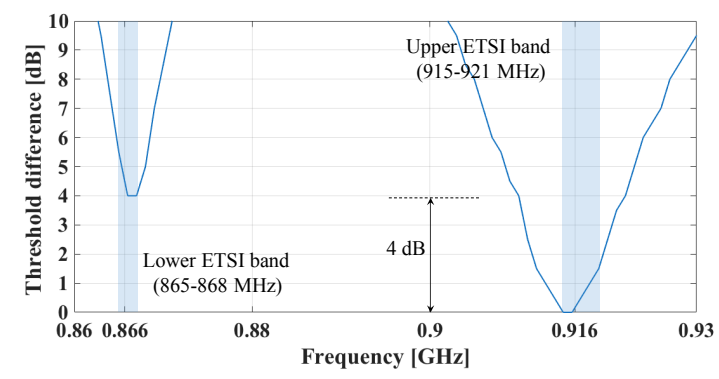

Fig. 6 Threshold difference of the Omni-ID Adept 850 tag in two ETSI bands. To ease comparison, all thresholds have been normalised to the threshold at $916 \mathrm{MHz}$

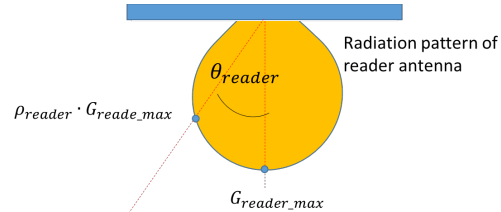

(a)

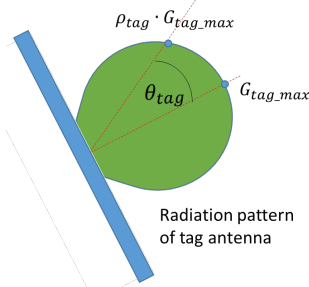

(b)

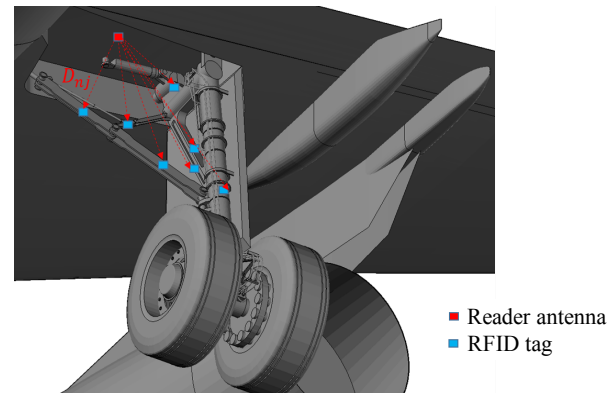

Fig. 7 System feasibility test model (a) the link model takes the angular dependence of both reader and tag antennas into account (b) a 3D model of aircraft landing gear. Not all reader antennas and tags are presented in the 3D model. The reader antenna presented here is similar to antenna location 5 shown in Fig. 3

requires at least operation across both the 868 and $915 \mathrm{MHz}$ bands [22].

One promising feature of the system would be the ability to update information on each tag. However, this requires write operations to the tags which typically requires an extra $5 \mathrm{~dB}$ reader transmitted power, according to the IC survey shown in Fig. 4. The two selected tags have been tested for both read and write sensitivity when a 32 -word (or 512 bits) user memory is read and written. It is found that reading 512 bits from user memory has the same sensitivity as reading the EPC in both cases. However, writing to the memory requires more power: $1.5 \mathrm{~dB}$ for Omni-ID tag, and $3.5 \mathrm{~dB}$ for HL tag. It is worth noting that the measured sensitivity difference for Omni-ID tag is not the same as the difference stated on the IC datasheet (see Table I). This is likely to be due to worst case values being listed in the datasheet, with practical performance likely to be better.

\section{SYSTEM FEASIBILITY TEST MODEL}

As mentioned the maximum system operating range occurs when reader and tag antennas are facing each other with their peak gains. However, for this application it is impossible to ensure that all tags are facing reader antennas with their peak gains, since the tags are placed on many different LG components with surfaces which are not in a common plane. As shown in Fig. 7 (a), if two antennas are not facing each other with their peak gains (the EM radiation is assumed to be generated from the antenna centres), the reader-to-tag distance must be shortened such that the power delivered to the tag is sufficient to turn on the tag IC: 


$$
P_{I C}=\frac{P_{\text {reader }, T} \cdot G_{\text {reader }, T, \text { max }} \cdot \rho_{\text {reader }} \cdot G_{\text {tag }, R, \text { max }} \cdot \rho_{\text {tag }} \cdot c^{2} \cdot X_{d} \cdot \tau}{(4 \pi f D)^{2} \cdot L_{d b}},
$$

where $\rho_{\text {reader }}$ and $\rho_{\text {tag }}$ are the gain penalties for reader and tag antennas due to their misalignment, and $D$ is the reader antenna to tag distance. Values for the gain penalties can be extracted from the measured tag and reader antenna radiation patterns, and the misalignment angles (i.e. $\theta_{\text {reader }}$ and $\theta_{\text {tag }}$ ) measured from the LG 3D model. By combining Eqns. 1 and 4, the system range of each reader antenna-tag pair $D$ can be expressed as:

$$
D=D_{\text {max }} \sqrt{\rho_{\text {reader. }} \rho_{\text {tag }}},
$$

where $D_{\max }$ is $2.8 \mathrm{~m}$ and $5.3 \mathrm{~m}$ for Omni-ID tag and $\mathrm{HL}$ tag respectively (as shown in Fig.5(b) the beamwidth of the reader antenna is wider than $90^{\circ}$ so the total radiated power must be lowered by $3 \mathrm{~dB}[21])$.

Considering the footprint of the reader antennas and available spaces in the wing bay and on the main fitting, 8 reader antenna locations, and 9 tag locations have been selected ( 4 tag locations MF1-MF4, are available for the main fitting, see Fig. 3) from a 3D aircraft model, as shown in Fig. 7 (b). By combining the antenna and tag characteristics with geometric information from the model, the required read range for each combination can be compared with the expected read range for the tag, antenna and geometry. The results are shown in Figs. 8 (a) and (b) for Omni-ID tag and HL tag, respectively. Within each bold cell, the upper cell represents the reader-to-tag distance measured from the 3D model, and the lower cell shows the distance estimated based on the radiating characteristics of the reader and tag antennas. If the value in the lower cell is greater, then the cell is highlighted in a colour representing the power margin (i.e. the square rooted term in Eq. 3 ) in $\mathrm{dB}$; white cells denote a negative power margin or combinations where tag reads are not expected to be possible. There are some tag and reader antenna locations that do not have a clear LoS, and for those tag-antenna pairs the model cannot be applied. The corresponding cells are highlighted in grey.

Due to its longer maximum read range, HL tag is expected to function better than Omni-ID in the system with greater power margins (i.e. colors are warmer). It allows more antenna options for successful reading of all LG components (the main fitting is considered to be detected if one of the four tags is detected). In addition, antennas 1 to 4 in the wing bay are expected to work better with the upper LG components, such as the lock stay and the side stays (see Fig. 3). They however are not expected to work well with the torque link damper and the axle, as the paths to these components are too long $(>2.2 \mathrm{~m})$ and are blocked by other LG components. In comparison, antennas 6 to 8 on the LG main fitting are close and have unblocked sights to the torque link damper and the axle, and as a result there should be no problem to read those two components.

Overall, antenna 5 is expected to perform the best. As shown in Figs. 3 and 7, antenna 5 is affixed to the top of the wing bay which is close to the main fitting and the upper LG components. Besides, its LoS to the torque link damper and the axle are not blocked by other LG components and thus is expected to detect them. (a)

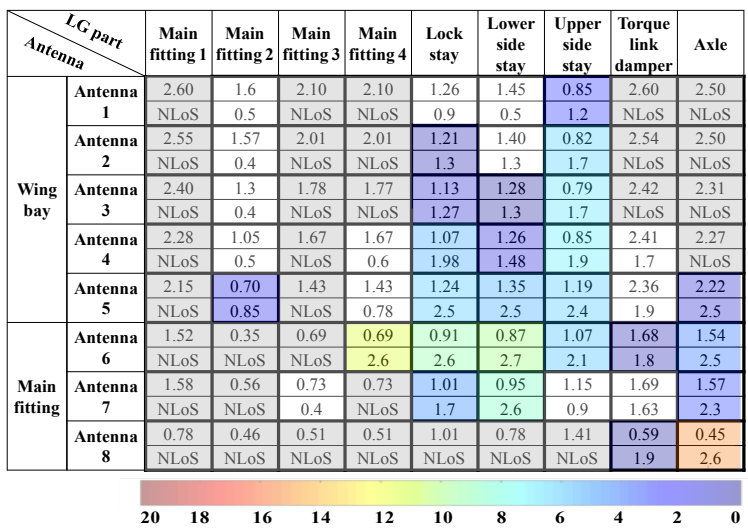

(b)

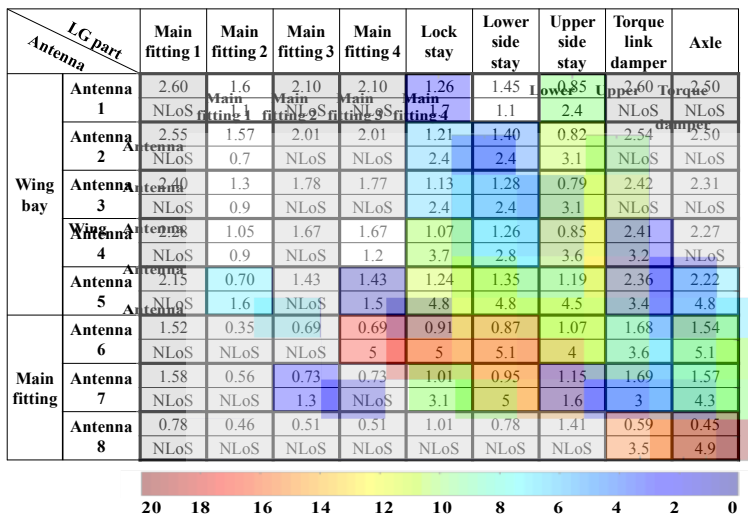

Fig. 8 System performance estimation based on radiating characteristics of (a) Omni-ID tag (b) HL tag. For both systems antenna 5 is expected to perform the best

It is worth noting that here the beam patterns of the reader and tag antennas are not considered to be distorted by the nearby objects (i.e. other LG components) or the shape of the attaching surface. However, in practice they will be and the effects are usually unpredictable without detailed EM simulation or experimentation.

\section{AIRCRAFT-LEVEL TEST RESULTS AND ANALYSES}

To determine if the system functions as the model predicts, system-level tests have been carried out in situ on an Airbus A320. The right-hand main landing gear was chosen for the initial system evaluation. A complete aircraft was required for tests due to the need to determine the potential impact of large metal structures of the wings and aircraft body which influence the RF propagation. The $\mathrm{A} 320$ was chosen due to the availability of a test aircraft and the large number of aircraft in service. However, the results are expected to be broadly transferable to other similar sized aircraft.

Fig. 9 shows the installed locations of RFID reader antennas and tags. Their spatial locations can be found in Fig. 3. The tags were temporarily attached onto the LG components using double-sided tape. In real applications, strong adhesive is required to maintain the attachment at extreme temperatures and offer superior protection against harsh environments. The 8 reader antennas were attached to the pipes running though the wing bay via cable ties, and affixed to the main fitting using double-sided tape. It is expected that in a final implementation, 


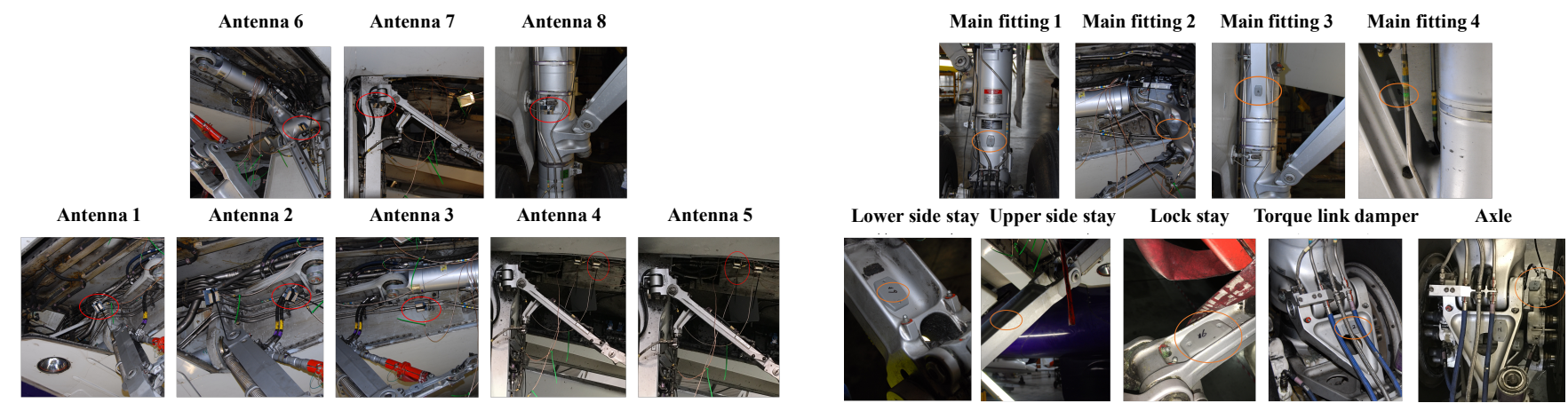

Fig. 9 Locations of reader antennas (left) and tags (right). Antennas 6 to 8 are located on the LG main fitting, and the rest antennas are located in the wing bay

modifications of the p-brackets which currently mount the pipes may also provide mountings for the antennas.

An Impinj R420 reader [23] was selected to test the system performance in the lower ETSI frequency band (865-868 MHz) due to its high output power of $31.5 \mathrm{dBm}$ which is sufficient to produce the maximum allowable transmitted power of 32.15 $\mathrm{dBm}$ EIRP (lowered by $3 \mathrm{~dB}$ due to antennas' wider beamwidths) via the $2.5 \mathrm{dBi}$ ceramic antennas. In addition, the reader has a high sensitivity is $-82 \mathrm{dBm}$ which compensates for the lack of antenna gain in the return link. The reader will be integrated into the DAQ and placed in the LG bay. Here for the

(a)

\begin{tabular}{|c|c|c|c|c|c|c|c|c|c|c|}
\hline \multicolumn{2}{|c|}{ 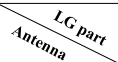 } & \begin{tabular}{|} 
Main \\
fitting 1
\end{tabular} & $\begin{array}{c}\text { Main } \\
\text { fitting 2 }\end{array}$ & $\begin{array}{c}\text { Main } \\
\text { fitting 3 }\end{array}$ & $\begin{array}{c}\text { Main } \\
\text { fitting 4 }\end{array}$ & $\begin{array}{l}\text { Lock } \\
\text { stay }\end{array}$ & \begin{tabular}{|c|} 
Lower \\
side \\
stay
\end{tabular} & \begin{tabular}{|l|} 
Upper \\
side \\
stay
\end{tabular} & \begin{tabular}{|c|} 
Torque \\
link \\
damper
\end{tabular} & Axle \\
\hline \multirow{5}{*}{$\begin{array}{c}\text { Wing } \\
\text { bay }\end{array}$} & \begin{tabular}{|c|} 
Antenna \\
1 \\
\end{tabular} & & 9 & 6 & 2 & 11 & 3 & 10 & & \\
\hline & \begin{tabular}{|c|} 
Antenna \\
2
\end{tabular} & & 12 & & 2 & 10 & & 12 & & \\
\hline & \begin{tabular}{|c|} 
Antenna \\
$\mathbf{3}$
\end{tabular} & & 17 & 3 & & 7 & & 12 & & \\
\hline & \begin{tabular}{|c|c} 
Antenna \\
4 \\
\end{tabular} & & 10 & 2 & 4 & 5 & 3 & 13 & & \\
\hline & \begin{tabular}{|c} 
Antenna \\
5
\end{tabular} & 1 & 14 & 8 & & 8 & & 12 & & 1 \\
\hline \multirow{3}{*}{$\begin{array}{l}\text { Main } \\
\text { fitting }\end{array}$} & \begin{tabular}{|c|} 
Antenna \\
6 \\
\end{tabular} & 7 & 18 & 8 & 1 & 2 & 8 & 10 & 0.5 & 1 \\
\hline & \begin{tabular}{|c|} 
Antenna \\
7 \\
\end{tabular} & 3 & 14 & 12 & 10 & 12 & 0.5 & 1 & & 1 \\
\hline & \begin{tabular}{|c|c} 
Antenna \\
8
\end{tabular} & 5 & 6 & 14 & 3 & & 7 & 1.5 & 17 & 7 \\
\hline
\end{tabular}

(b)

\begin{tabular}{|c|c|c|c|c|c|c|c|c|c|c|}
\hline \multicolumn{2}{|c|}{ 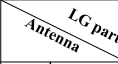 } & $\begin{array}{c}\text { Main } \\
\text { fitting 1 }\end{array}$ & \begin{tabular}{|c} 
Main \\
fittting 2
\end{tabular} & $\begin{array}{c}\text { Main } \\
\text { fitting 3 }\end{array}$ & $\begin{array}{c}\text { Main } \\
\text { fitting 4 }\end{array}$ & $\begin{array}{l}\text { Lock } \\
\text { stay }\end{array}$ & \begin{tabular}{|c|} 
Lower \\
side \\
stay
\end{tabular} & $\begin{array}{l}\text { Upper } \\
\text { side } \\
\text { stay }\end{array}$ & \begin{tabular}{|c|} 
Torque \\
link \\
damper
\end{tabular} & Axle \\
\hline \multirow{5}{*}{$\begin{array}{l}\text { Wing } \\
\text { bay }\end{array}$} & \begin{tabular}{|c|} 
Antenna \\
1 \\
\end{tabular} & 1 & 12 & 1 & 7 & 7 & 8 & 2 & 7 & \\
\hline & \begin{tabular}{|c} 
Antenna \\
$\mathbf{2}$ \\
\end{tabular} & 7 & 9 & 9 & 18 & 9 & 14 & 14 & 6 & \\
\hline & \begin{tabular}{|c|} 
Antenna \\
3 \\
\end{tabular} & 1 & 18 & 5 & 13 & 4 & 6 & 8 & 0.5 & \\
\hline & \begin{tabular}{|c|} 
Antenna \\
4 \\
\end{tabular} & 5 & 3 & 9 & 12 & 14 & 14 & 14 & 5 & \\
\hline & \begin{tabular}{|c|} 
Antenna \\
5
\end{tabular} & 2 & 13 & 2 & 11 & 17 & 5 & 6 & 4 & 7 \\
\hline \multirow{3}{*}{$\begin{array}{c}\text { Main } \\
\text { fitting }\end{array}$} & \begin{tabular}{|c|} 
Antenna \\
6 \\
\end{tabular} & 16 & 17 & 17 & 9 & 15 & 17 & 0.5 & 1 & 1 \\
\hline & \begin{tabular}{|c|} 
Antenna \\
7 \\
\end{tabular} & & 20 & 13 & 16 & 13 & 7 & 6 & 5 & 1 \\
\hline & \begin{tabular}{|c|} 
Antenna \\
8 \\
\end{tabular} & 12 & 3 & 14 & 16 & 14 & 10 & 4 & 13 & 6 \\
\hline
\end{tabular}

Fig. 10 Aircraft-level test results of the (a) Omni-ID tag and (b) HL tag based systems. The tag (LG component) can be detected by the corresponding antenna if there is a value in the cell. The colored and white cells represent the LG components at which the tag are, and are not expected to be detected by the corresponding reader antenna respectively, according to the predictions shown in Fig.8; The grey cells represent those LG components at which the tag does not have a clear LoS with the corresponding antenna ease of testing the reader was placed in the wing bay close to the LG leg assembly as shown in Fig. 3.

Fig. 10 shows the RFID system performance for the two tags in terms of the achieved Tx power margin with respect to the maximum EIRP. Numbers in the cells show the power margin achieved by that antenna and tag combination. To compare the test results with those predicted by the model, the same coloring used in Fig. 8 is applied to the tables. It can be seen that most reader antenna-tag pairs previously predicted to be functional are indeed functional, but there are deviations in system margins which are expected as the results of reflections and distortions in antenna radiation patterns caused by nearby and attached metal objects. The same explanation can be used to explain why tags on the lower side stays (Fig. 9 (right)) cannot be detected by antennas 3 and 5 , even if the physical distances are only around $1.3 \mathrm{~m}$ (see corresponding values in Fig. 8 (a)).

As expected, HL tag outperforms Omni-ID tag with more functional reader antenna-tag pairs, as well as higher system margins. However, it is also shown that for certain reader antenna-tag pairs the Omni-ID tag performs better. This suggests that the surrounding and attached objects have different effects on the two tags with different antenna designs.

For those reader antenna-tag pairs without a direct LoS (i.e. cells in grey), if the physical distance is long (e.g. $>2 \mathrm{~m}$ ), as predicted, they are not functional in practice. An example is the tags on the LG axle when read by antennas 1 to 4 in the wing bay. However, one exception is the HL tag on main fitting 1 (MF1, see Figs. 3 and 9). None of the reader antennas has a LoS with this tag location, especially for antenna 1 (see Figs.3 and 8) which has the longest physical distance of around $2.6 \mathrm{~m}$. In addition, the tag is facing away from the LG which adds difficulty in being read. One explanation for the successful read is that after multiple reflections, reader signals at the tag location add constructively and hence can power the tag. That is possible, but a more feasible explanation is that the tag has been read due to the induced E-field on the main fitting. It has been shown in [24] that when placed close to an antenna, the E-field distribution above a long thin metal bar is stronger than that of the free space, and hence it is possible to read a tag with lower reader transmitted power. In our case, the LG main fitting (see Fig. 3) is just a long conductive tube with one end illuminated by several nearby antennas. Although the induced current on the main fitting is likely to spread in two directions, since metals are good conductors the E-field at the tag location can still be higher 
(a)

\begin{tabular}{|c|c|c|c|c|c|c|c|c|c|c|}
\hline & $\begin{array}{c}\text { Main } \\
\text { fitting 1 }\end{array}$ & $\begin{array}{c}\text { Main } \\
\text { fitting 2 }\end{array}$ & $\begin{array}{c}\text { Main } \\
\text { fitting 3 }\end{array}$ & $\begin{array}{c}\text { Main } \\
\text { fitting 4 }\end{array}$ & $\begin{array}{l}\text { Lock } \\
\text { stay }\end{array}$ & \begin{tabular}{|l} 
Lower \\
side \\
stay
\end{tabular} & \begin{tabular}{|l} 
Upper \\
side \\
stay
\end{tabular} & \begin{tabular}{|c} 
Torque \\
link \\
damper
\end{tabular} & Axle \\
\hline \multirow{5}{*}{$\begin{array}{c}\text { Wing } \\
\text { bay }\end{array}$} & $\begin{array}{c}\text { Antenna } \\
1\end{array}$ & & -39 & -54 & -57 & -51 & -56 & -49 & & \\
\hline & $\begin{array}{c}\text { Antenna } \\
\mathbf{2}\end{array}$ & & -33 & & -53 & -52 & & -49 & & \\
\hline & \begin{tabular}{|c} 
Antenna \\
3 \\
\end{tabular} & & -35 & -56 & & -54 & & -45 & & \\
\hline & \begin{tabular}{|c|} 
Antenna \\
4 \\
\end{tabular} & & -38 & -42 & & -54 & -56 & -38 & & \\
\hline & $\begin{array}{c}\text { Antenna } \\
5\end{array}$ & -56 & -48 & -55 & & -56 & & -50 & & -69 \\
\hline \multirow{3}{*}{$\begin{array}{l}\text { Main } \\
\text { fitting }\end{array}$} & \begin{tabular}{|c|} 
Antenna \\
6 \\
\end{tabular} & -48 & -33 & -55 & -56 & -59 & -48 & -48 & -70 & -59 \\
\hline & \begin{tabular}{|c} 
Antenna \\
7 \\
\end{tabular} & -51 & -47 & -60 & -55 & -51 & -58 & -54 & & -59 \\
\hline & \begin{tabular}{|c} 
Antenna \\
8
\end{tabular} & -55 & -52 & -51 & -57 & & -53 & -62 & -44 & -52 \\
\hline
\end{tabular}

(b)

\begin{tabular}{|c|c|c|c|c|c|c|c|c|c|c|}
\hline \multicolumn{2}{|c|}{ 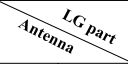 } & $\begin{array}{c}\text { Main } \\
\text { fitting } \\
1\end{array}$ & $\begin{array}{c}\begin{array}{c}\text { Main } \\
\text { fitting } \\
2\end{array} \\
\end{array}$ & $\begin{array}{c}\text { Main } \\
\text { fitting } \\
3\end{array}$ & $\begin{array}{c}\text { Main } \\
\text { fitting } \\
4\end{array}$ & $\begin{array}{l}\text { Lock } \\
\text { stay }\end{array}$ & $\begin{array}{l}\text { Lower } \\
\text { side } \\
\text { stay }\end{array}$ & $\begin{array}{l}\text { Upper } \\
\text { side } \\
\text { stay }\end{array}$ & \begin{tabular}{|c|} 
Torque \\
link \\
damper
\end{tabular} & Axle \\
\hline \multirow{5}{*}{$\begin{array}{l}\text { Wing } \\
\text { bay }\end{array}$} & $\begin{array}{c}\text { Antenna } \\
1\end{array}$ & -71 & -49 & -72 & -59 & -66 & -64 & -61 & -62 & \\
\hline & \begin{tabular}{|c|} 
Antenna \\
2 \\
\end{tabular} & -57 & -54 & -59 & -45 & -43 & -52 & -49 & -50 & \\
\hline & \begin{tabular}{|c|} 
Antenna \\
3 \\
\end{tabular} & -58 & -50 & -69 & -59 & -67 & -66 & -55 & -68 & \\
\hline & \begin{tabular}{|c|} 
Antenna \\
4 \\
\end{tabular} & -65 & -66 & -63 & -59 & -45 & -53 & -48 & -61 & \\
\hline & \begin{tabular}{|c} 
Antenna \\
5 \\
\end{tabular} & -70 & -59 & -71 & -60 & -53 & -60 & -57 & -66 & -65 \\
\hline \multirow{3}{*}{$\begin{array}{c}\text { Main } \\
\text { fitting }\end{array}$} & \begin{tabular}{|c} 
Antenna \\
6 \\
\end{tabular} & -57 & -54 & -54 & -64 & -57 & -53 & -70 & -67 & -69 \\
\hline & \begin{tabular}{|c|} 
Antenna \\
7 \\
\end{tabular} & & -47 & -57 & -56 & -59 & -59 & -52 & -64 & -68 \\
\hline & $\begin{array}{c}\text { Antenna } \\
8\end{array}$ & -64 & -70 & -62 & -60 & -60 & -64 & -61 & -60 & -70 \\
\hline
\end{tabular}

Fig. 11 Reader received power from (a) Omni-ID tags (b) HL tags at their thresholds. In each table, the highlighted cells represent the reader received power from that tag location at which is not detectable for the other tag

than the tag E-field threshold and hence the tag can be read. The same theory can be used to explain why tags (both Omni-ID and HL tags) on main fitting 2 can be read by all antennas, and why antenna 8 on the main fitting can read almost all the tags on the upper sections of the landing gear with very high system margins, even if its overall radiation is directed away from them.

Since the model assumes that the system is downlink limited, i.e. the system fails to work because of the lower reader incident power at the tag. To check the validity of this assumption, the reader received power at each antenna, from each tag at its power threshold is recorded. The results are shown in Fig. 11.

In each table, the highlighted cells represent the reader received power from that tag location at which the other type of tag is not detectable. Clearly there are more failures to read the Omni-ID tags (hence more LG components) than HL tags. However, it can be seen that most of the reader received powers at these locations vary between -50 to $-65 \mathrm{dBm}$, with the worst $-71 \mathrm{dBm}$ located at main fitting 1 , read by antenna 1 . The reader used in the tests has a sensitivity of $-82 \mathrm{dBm}$, and therefore the system can tolerate at least $15 \mathrm{~dB}$ less tag backscattered power (note that the numbers in the tables are the reader received powers at tag thresholds, i.e. the values will be greater when taking the power margin into account). However, if we have a look at the system margins for HL tags at which Omni-ID tags were not readable (see the cells in Fig. 10 (b) that are highlighted in Fig. 11 (b)), most margins are less than $7 \mathrm{~dB}$, which is close to the tag sensitivity difference as mentioned previously. Therefore, the Omni-ID tag based system should still be downlink limited. Since the tag read requires $4 \mathrm{~dB}$ less power in the upper ETSI band (see Fig. 6) when the system moves to the upper ETSI band, with an extra $3 \mathrm{~dB}$ power allowance the missing Omni-ID tags may be detectable.

Although the use of more reader antennas provides greater system margins, it also increases the complexity of the system design. Fig. 12 shows results of different system performances with up to 2 reader antennas.

Tables (a) and (b) shown in Fig. 12 also list the missing LG components in the system when Omni-ID tags and HL tags are used respectively (here the main fitting is considered to be detected if any 1 out of 4 tags is detected). The diagonal cells

(a)

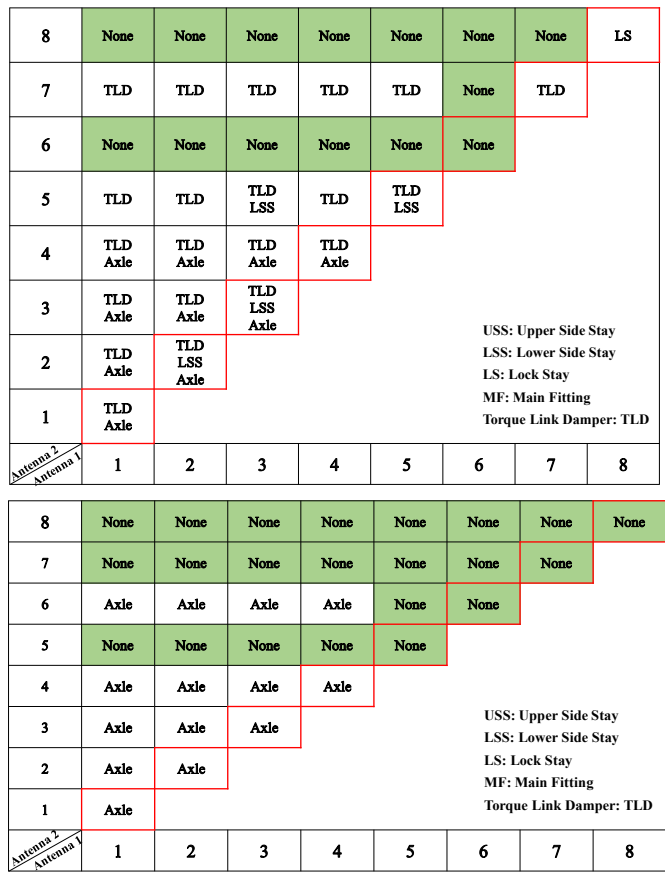
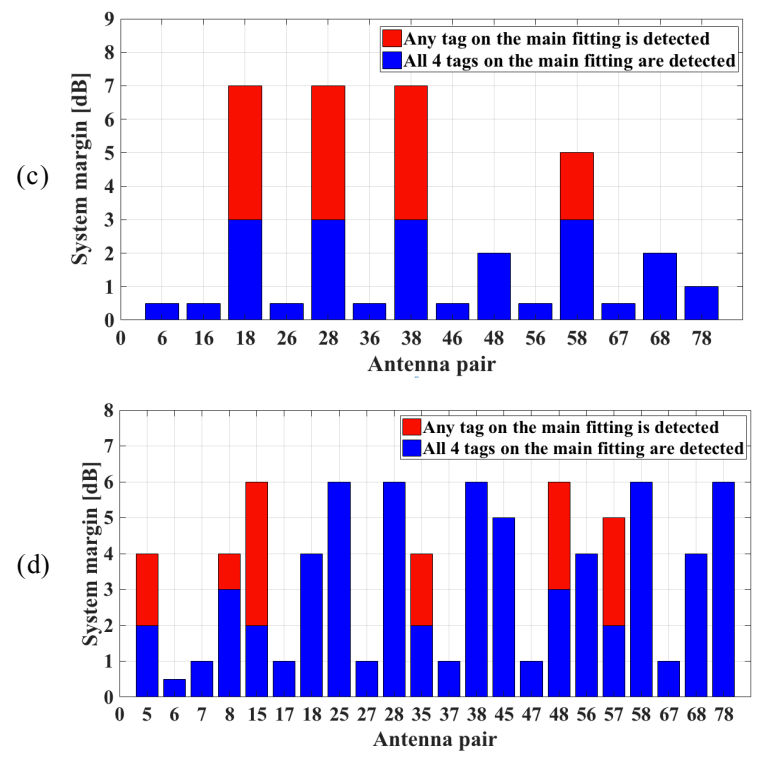

Fig. 12 Different system performances with up to 2 reader antennas (a) missing LG components in the Omni-ID tag-based system (b) missing LG components in the HL tag-based system (c) system margins for the Omni-ID tag-based system (d) system margins for the HL tag-based system 
(framed red) list the non-detected LG components when only a single antenna is used in monostatic mode. If all the LG components are detected, the cell is colored in green. As in the results presented in previous sections, the HL tag-based system performs better due to the tag's longer range. All the LG components can be detected using only antenna 5 in the wing bay, or antennas 6 to 8 on the main fitting. The LG axle is shown to be the only undetected LG component since its distance to all antennas except 1 to 4 in the wing bay are too long and blocked by some LG components. In addition, the tag's radiation pattern is expected to be distorted by the nearby wheel (which has metal wire in the tyre) and brake.

For the Omni-ID tag-based system, it is shown that only using antenna 6 on the main fitting can detect all the tags on the LG components. However, in practice, this location (see Fig. 9) is not ideal due to the curved surface which the antenna must be attached to and its exposure to high airflow. Compared with other antennas, antenna 8 on main fitting (see Figs. 3 and 9) only missed the tag on the lock stay. Furthermore, this location is more promising than other antennas on the main fitting, since: 1) it is closer to the tags on the torque link damper and the axle, and 2) the landing gear door can be used to strengthen the antenna's attachment to the main fitting. It is therefore expected that using antenna 8 in conjunction with one antenna in the wing bay, such as antenna 5 to form a 2-antenna system could offer a much better system performance.

Figs. 12 (c) and (d) show the system margins for the OmniID tag- and HL tag-based systems respectively. In case we want all tags, including all 4 tags on the main fitting to be detected, the maximum system margin for a single antenna system is 0.5 $\mathrm{dB}$ for the Omni-ID tag-based system and $3 \mathrm{~dB}$ for HL tag-based system. Such a margin would allow the Omni-ID tag-based system to read (and write if the system operates in the upper ETSI band) configuration data of the LG components as well as sensor data; however, since the margin is lower than the difference between the tag's read and write sensitivities (i.e. 3.5 $\mathrm{dB})$, it is not expected that write operations will be possible in the lower ETSI band. Therefore, the dynamic information must be stored first on the reader and later transferred to the tags using a handheld reader when major maintenance occurs. In contrast, for the HL tag-based system, the $3 \mathrm{~dB}$ margin would allow both read and write operations to the tags and hence information on the tags can be updated anytime when necessary. In addition, if we only require a single tag to be attached to the main fitting, there is no margin change for the Omni-ID tag-based system, but for the HL tag-based system, the margin increases to at least $4 \mathrm{~dB}$. The only drawback for this system is the lower memory capacity on each tag.

For the 2-antenna-based systems, when HL tags are used the maximum margin is $6 \mathrm{~dB}$, achievable by multiple antenna pairs. It is ideal to locate the reader antennas in the wing bay for the ease of cable routing. Therefore, antennas 1 and 5, or 2 and 5 are promising options. For the system that ultilises Omni-ID tags, a maximum margin of $3 \mathrm{~dB}$ can be achieved by multiple antenna pairs. However, only read operations can be carried out in the lower ETSI band. If we only attach 1 tag to the main fitting, the system margin can be boosted to $7 \mathrm{~dB}$ with antenna pairs $(1,8)$, $(2,8)$, and $(3,8)$. Such a margin would allow write operations to the tags, as well as some resilience against degradations caused by different operating environments (e.g. low/high temperature). As shown in Fig. 4, the sensitivity difference between Q-star 2A and MB97R7051 is around $8 \mathrm{~dB}$. It is therefore believed that by a proper antenna design (i.e similar radiating characteristics to Omni-ID tag's antenna), the system would allow a tag with a maximum of 64 kbyte of memory to be attached to each LG component when it is operating in the upper ETSI band with a system margin of $6 \mathrm{~dB}$ (i.e $7 \mathrm{~dB}$ margin in the lower ETSI band $+3 \mathrm{~dB}$ more power in the upper ETSI band $+4 \mathrm{~dB}$ tag sensitivity difference in the two bands $-8 \mathrm{~dB}$ IC sensitivity difference). Since the MB97R7051 IC uses FeRAM, the read and write sensitives are identical, so writing to the tag would also be possible.

\section{CONCLUSIONS}

This paper has described how passive RFID can be used for the landing gear health monitoring, and has reported the performance of a passive RFID-based configuration control system for landing gear components in the lower ETSI frequency band. A model based on the radiating characteristics of both reader and tag antennas has been developed to determine the system feasibility prior to aircraft level tests. The practical test results indicate that the model can be used to predict the readability of a tag when there exists a clear LoS to the reader antenna. However, since the environment is so complex, the relative system margin cannot be accurately predicted. The positive/negative impacts of the inevitable EM induction on the metal structures due to a nearby antenna (can be analysed using EM simulation software) on the tag must be considered (positive impacts were found in the aircraft level tests, and thus metals are not always troublesome for UHF RFID applications).

Depending on the memory requirement from the tags (i.e. 8 kbits or 64 kbits), it is shown that the system can wirelessly collect and update the LG components' data stored on the RFID tags with up to 2 reader antennas in the lower ETSI band. It is expected that when a reader which can transmit upper ETSI frequencies and greater powers becomes available, the system would allow more system margin, and therefore data up to $64 \mathrm{k}$ bytes to be associated with each LG components.

\section{ACKNOWLEDGMENT}

This work is partly supported by the Innovate UK through the LAGEMOSYS project.

\section{REFERENCES}

[1] K. Finkenzeller, RFID handbook. Chichester, England: Wiley, 2003

[2] P. D. Devries, "The State of RFID for Effective Baggage Tracking in the Airline Industry," International Journal of Mobile Communications, vol. 6, no. 2, pp. 151-164, 2008

[3] J. Hendry, P. Ryan and N. Bondarenco. (2010 November). Baggage Improvement Program, US Airways Case Study Boston Logan International Airport (BOS) [Online]. Available: http://www.iata.org/whatwedo/stb/bip/Documents/bip-us-airways-casestudy-2010.pdf

[4] C. R. Medeiros, J. R. Costa and C. A. Ferandes, "Passive UHF RFID Tag for Airport Suitcase Tracking and Identification," IEEE Antennas and Wireless Propagation Letters, vol. 10, pp. 123-126, March, 2011

[5] A. Price, "RFID Business Case for Baggage Tagging", IATA, 2007

[6] "RFID Integrated solutions System Optimizes Maintenance Efficiency," The Boeing Commercial Aeromagazine, 2012

[7] "Radio Frequency Identification (RFID), Airbus Business Radar", Airbus Flight Airworthiness Support Technology (FAST) 47, January 2011 
[8] M. Robert (2004, May 6). "Boeing, Airbus Team on Standards," RFID Journal [Online]. Available: http://www.rfidjournal.com/articles/view?934 [Accessed: Jan. 05, 2016]

[9] S. Bradfield, S. Sarkar, C. McColl and N. Phan, "Architecture for Dynamic Component Life Tracking in an Advanced HUMS, RFID, and Direct Load Sensor Environment," 2009

[10] Attilio Arcari , "Improving Passive RFID Tag Performance: Application to Rotorcraft Dynamic Component Tracking," HUMS Conference, 2013

[11] SINGH, A. et al., "Lessons Learned from the Structural Life Tracking of Rotorcraft Dynamic Components," 6th European Workshop on Structural Health Monitoring, 2012

[12] Geoffrey Holmes, Pia Sartor, Stephen Reed, Paul Southern, Keith Word en and Elizabeth Cross "Prediction of landing gear loads using machine learning techniques," Structural Health Monitoring Vol 15, Issue 5, pp. $568-582,2016$

[13] ATA Spec 2000 Chapter 9, Automated Identification and Data Capture (AIDC), 2016

[14] "Fujitsu, Expand the Possibilities of Your RFID Tags with FRAM," Fujitsu, [Online]. Available: https://www.fujitsu.com/cn/Images/FRAM_RFID_LSI_2012.11_EN.pdf [Accessed: 19 January 2017]

[15] www.omni-id.com

[16] www.hl-rfidtag.com

[17] Quanray Electronics, "Qstar-2A Brief Spec," RFID IC datasheet, [Online]. Available: http://www.quanray.com/en/UpLoadFile/Product/ 20117461305681.pdf [Accessed: 17 January 2016]

[18] Impinj, "Impinj Monza X-8k Dura Data Sheet," IPJ-P6005-X2AT datasheet, 2015

[19] P. V. Nikitin, K. V. S. Rao and S. Lam, "UHF RFID TAG CHARACTERISATION: OVERVIEW AND STATE-OF-THE-ART," AMTA, Seattle, WA, October 2012

[20] Passive RFID Tags Intended for Aircraft Use, SAE AS 5678A, 2015

[21] ETSI EN 302 208-1, Electromagnetic compatibility and Radio spectrum Matters (ERM); Radio Frequency Identification Equipment operating in the band $865 \mathrm{MHz}$ to $868 \mathrm{MHz}$ with power levels up to $2 \mathrm{~W}$ and in the band $915 \mathrm{MHz}$ to $921 \mathrm{MHz}$ with power levels up to $4 \mathrm{~W}$; Part 1: Technical requirements and methods of measurement, European Standard REN/ERM-TG34-260, 2015

[22] GS1, "Regulatory status for using RFID in the EPC Gen 2 band (860-960 MHz) of the UHF spectrum," GS1, 2014. [Online]. Available: www.gs1.org. [Accessed: Jan. 17, 2016]

[23] Imping, "Speedway RAIN RFID Readers," [Online]. Available: http://www.impinj.com/products/readers/speedway-revolution/ [Accessed: 17 January 2016]

[24] S. Yang et al., "RFID System Exploiting Large Metal Objects as Near Field UHF RFID Antennas," in IEEE International Conference on RFID, pp. $96-102,2017$ 